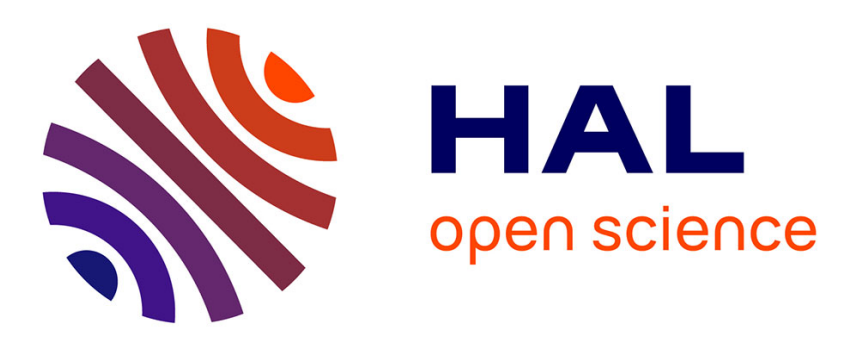

\title{
Liens territoriaux, liens sociaux : le territoire, support ou prétexte?
}

Stéphanie Vermeersch

\section{To cite this version:}

Stéphanie Vermeersch. Liens territoriaux, liens sociaux: le territoire, support ou prétexte?. Espaces et sociétés (Paris, France), 2006, Les lieux des liens sociaux, 126, pp.53-68. 10.3917/esp.126.0053 . halshs-01216679

\section{HAL Id: halshs-01216679 \\ https://shs.hal.science/halshs-01216679}

Submitted on 16 Oct 2015

HAL is a multi-disciplinary open access archive for the deposit and dissemination of scientific research documents, whether they are published or not. The documents may come from teaching and research institutions in France or abroad, or from public or private research centers.
L'archive ouverte pluridisciplinaire HAL, est destinée au dépôt et à la diffusion de documents scientifiques de niveau recherche, publiés ou non, émanant des établissements d'enseignement et de recherche français ou étrangers, des laboratoires publics ou privés. 


\section{LIENS TERRITORIAUX, LIENS SOCIAUX : LE TERRITOIRE, SUPPORT OU PRÉTEXTE?}

\section{Stéphanie Vermeersch}

\section{ERES | « Espaces et sociétés »}

$2006 / 3 \mathrm{n}^{\circ} 126$ | pages 53 à 68

ISSN 0014-0481

ISBN 2749206421

Article disponible en ligne à l'adresse :

http://www.cairn.info/revue-espaces-et-societes-2006-3-page-53.htm

\section{!Pour citer cet article :}

Stéphanie Vermeersch, «Liens territoriaux, liens sociaux : le territoire, support ou prétexte? », Espaces et sociétés 2006/3 ( $\left.\mathrm{n}^{\mathrm{o}} 126\right)$, p. 53-68.

DOI 10.3917/esp.126.0053

Distribution électronique Cairn.info pour ERES.

(C) ERES. Tous droits réservés pour tous pays.

La reproduction ou représentation de cet article, notamment par photocopie, n'est autorisée que dans les limites des conditions générales d'utilisation du site ou, le cas échéant, des conditions générales de la licence souscrite par votre établissement. Toute autre reproduction ou représentation, en tout ou partie, sous quelque forme et de quelque manière que ce soit, est interdite sauf accord préalable et écrit de l'éditeur, en dehors des cas prévus par la législation en vigueur en France. Il est précisé que son stockage dans une base de données est également interdit. 


\title{
1006
}

\section{Liens territoriaux, liens sociaux : le territoire, support ou prétexte?}

\section{Stéphanie Vermeersch}

\begin{abstract}
L es recherches sur les processus sociaux à l'œuvre en milieu urbain soulignent combien les couches moyennes, qui investissent les quartiers anciens centraux, sont portées par une idéologie de la mixité sociale, qu'elles contredisent toutefois par des pratiques sociales valorisant l'entre-soi (Bacqué, Fol, Lévy, 1998). Les associations de quartier sont alors susceptibles de les aider, entre autres, à résoudre cette opposition : en leur permettant de se mettre en lien avec les couches populaires et ainsi de concrétiser le mythe du « quartier village » auquel elles sont attachées (Mehl, 1982), elles font du quartier un lieu de liens apparemment ouverts et non-discriminants. En articulant nombre de leurs discours et de leurs activités, festives ou militantes, autour des notions de « vivre ensemble », de « mixité sociale », elles semblent se positionner à rebours du fractionnement social de l'espace urbain et désireuses de construire le territoire comme lieu de liens sociaux pacifiés. L'espace associatif constitue-t-il un vecteur de construction de liens sociaux territoriaux, et quelle est alors la nature de ces derniers ?
\end{abstract}

Stéphanie Vermeersch, sociologue, chargée de recherche CNRS, UMR LOUEST, auteur d'une thèse sur le bénévolat; travaille actuellement sur les discours des couches urbaines moyennes. stephanie.vermeersch@paris-valdeseine.archi.fr 
Dès les années 1970 et l'essor des mobilisations habitantes locales contre les opérations d'aménagement et de rénovation urbaines, les travaux sur le fait associatif soulignent son rôle dans la création et l'entretien de liens sociaux locaux (Ion, Micoud, Nizey, 1979 ; Dagnaud, 1979). Mais il apparaît que ces sociabilités sont largement ségrégées et que les liens tissés sur la base de l'appartenance territoriale, quand ils sont médiatisés par l'appartenance associative, sont loin de se distribuer au hasard au sein de la population du quartier (Benoit-Guilbot, 1986). Mais les recherches contemporaines sur les associations d'habitants intègrent peu d'analyses en termes d'acteurs collectifs et de groupes sociaux (Ion, Gillio, 2001). La problématique du lien territorial se trouve alors en partie tronquée : des lieux urbains sont investis, appropriés, défendus et fêtés, mais de quels types de liens sont-ils alors les supports?

Cet article entend poser la question de l'impact de l'appartenance à un territoire spatialement défini sur les formes de liens sociaux susceptibles de se nouer, ou non, entre les habitants. Il s'appuie sur l'hypothèse que les associations en sont l'une des déclinaisons et veut analyser comment se jouent, sur la base du rapport au quartier, les relations entre les membres d'une association de quartier, mais également entre eux et d'autres habitants rencontrés dans le cadre des actions organisées par l'association. Il se fonde sur une importante enquête de terrain, menée sur trois ans au sein d'une association de quartier.

\section{MÉTHODE DE RECHERCHE}

Le travail de thèse portait sur la comparaison de deux engagements bénévoles différents, l'un caritatif et l'autre comme militant de quartier, afin de cerner les similitudes engendrées par le caractère bénévole de l'action. L'enquête de terrain a couplé observation participante et entretiens. Je suis donc entrée au bureau de l'association de quartier et ai participé à l'essentiel des actions engagées. Un carnet de bord a été tenu qui constitue une grande partie du matériau utilisé ici. Les entretiens, réalisés avec les membres les plus actifs (une quinzaine), visaient à saisir le sens donné par les militants à leur engagement et notamment la façon dont ils l'envisageaient à l'égard de leur rapport au quartier.

Ces terrains ont été l'occasion d'analyser comment, dans un contexte social de recompositions institutionnelles et de valorisation de l'autonomie individuelle, l'engagement associatif est un lieu possible du travail identitaire en autorisant simultanément l'individualisation et la participation. Les moteurs de l'engagement que sont l'éthique et le plaisir sont en effet structurés d'une part par l'idéal du moi contemporain tel que décrit par Norbert Elias, impérativement autonome et singulier, d'autre part par une volonté et une nécessité tout aussi fortes d'intégration à des collectifs et de mise en lien avec le monde. Vermeersch, 2004. 
Ce travail a permis de souligner qu'au-delà de l'efficacité et de l'utilité d'un certain nombre d'interventions associatives, il convenait d'être prudent quant à la capacité du monde associatif à créer des liens entre ses membres et les destinataires de l'action. La construction de liens parfois très intenses (Bidart, 1988) entre les membres de l'association peut s'opérer au détriment de l'ouverture souhaitée (Vermeersch, 2002, 2004). Nous voudrions ici repenser cette conclusion dans le cadre de la coexistence de groupes sociaux différents : on peut s'interroger sur la capacité du lien territorial, médiatisé par une association de quartier, à transcender la différenciation sociale des modalités d'investissement du quartier ainsi que des sociabilités. Il ne suffit pas d'avoir des voisins pour « voisiner» (Héran, 1987) et la communauté de résidence n'est pas garante de l'établissement de liens sociaux. Pourtant, les associations d'habitants sont volontaristes et cherchent à multiplier les occasions de «vivre ensemble » : lors de ces moments militants ou festifs, comment le territoire commun intervient-il dans les différentes formes de liens qui se nouent?

Le quartier étudié, «Plaisance Pernety », se situe au sud du XIVe arrondissement de Paris, délimité par l'avenue du Maine à l'est, le boulevard Brune au sud et la rue Vercingétorix à l'ouest. Moins connu que le « triangle du XIVe »(Chalvon, Claverie, 1984) autour de la rue Daguerre, il est nettement moins embourgeoisé que l'ensemble de l'arrondissement : on y compte trois fois moins de cadres et de professions intellectuelles supérieures $(12,4 \%$ pour $38,8 \%)$, presque trois fois plus d'ouvriers $(12,4 \%$ pour $5,7 \%)$, et quasiment le double d'employés (38\% d'employés pour $20,2 \%{ }^{1}$ ). Les deux îlots situés au nord du quartier, plus centraux, comptent trois fois plus de cadres et professions intellectuelles supérieures que les deux îlots périphériques situés aux alentours de la Porte de Vanves, composés essentiellement de grands ensembles de logements sociaux. L'association étudiée, Urbanisme et Démocratie, agit au sein du quartier depuis 1993 et s'est donné pour objet de « faire participer les citoyens aux décisions qui entraînent une modification de leur cadre de vie, de défendre la qualité de vie et de veiller à la préservation de la diversité sociale, économique et culturelle ». Elle œuvre dans l'ensemble du quartier, mais l'essentiel de ses militants réside dans la partie nord. Héritière des grandes luttes des années 1970 qui secouèrent le quartier (Cherki, Mehl, 1979), elle est, par ses thèmes et ses répertoires d'action, ainsi que par sa population, tout à fait typique des associations de quartier parisiennes contemporaines. Largement composée de membres des classes moyennes et supérieures, elle investit simultanément le champ d'une « convivialité » destinée à faire se rencontrer «tous » les habitants du quartier (fêtes et repas de quartier, projection en plein air, expositions...) et celui 
d'un militantisme autour des questions d'aménagement urbain et de démocratie. Ses préoccupations sont à la fois environnementales (densification du bâti, voies de circulation rapide, bruit...), sociales (logements sociaux, pension de famille...) et participatives (conseils de quartier, affichage associatif, café associatif...).

Nous voudrions poser ici la question des sociabilités territorialisées, en émettant l'hypothèse que la ségrégation sociale des liens sociaux urbains est aujourd'hui masquée par le déploiement d'une idéologie de l'ouverture sociale prenant le territoire comme prétexte.

\section{QUAND LE TERRITOIRE EST CENSÉ FAIRE LIEN}

Les militants de l'association de quartier étudiée présentent des caractéristiques sociales, politiques et culturelles susceptibles d'en faire des héritiers de ceux que Monique Dagnaud a pu appeler, dans les années 1970-1980, la «classe d'alternative » (Dagnaud, 1981). Il s'agit largement de membres des couches moyennes et supérieures, dotés d'un niveau culturel élevé, de formation universitaire, exerçant des professions liées aux milieux intellectuels, à l'enseignement, à l'animation, au travail social et à la santé...

Critiques de la société de consommation individualiste, attentifs à leur cadre de vie, intéressés par la création de projets alternatifs de vie à l'échelle des quartiers, ils militent pour développer une vie locale riche en échanges sociaux, initiée et gérée par les habitants eux-mêmes, autour d'équipements associatifs, participatifs et culturels. Les thèmes communs aux membres de la « classe d'alternative » irriguent largement leur réflexion et leur action : convivialité, démocratie directe, participation, autogestion. Intéressés par le politique, ils votent principalement pour les partis de gauche, avec une préférence pour la gauche non-socialiste et les écologistes. Là, se situe peut-être une différence avec leurs aînés des années 1970 et 1980 : c'est en partie une désillusion, voire une désespérance à l'égard de la politique, qui les amène vers une action locale, présentant l'avantage d'être efficace, concrète et dont on peut constater les résultats. L'action militante est ainsi ramenée à ses conditions de possibilité, d'efficacité et de satisfaction. Leurs motivations, pour n'être pas dépolitisées, sont pragmatiques, typiques du militantisme contemporain (Ion, Franguiadakis, Viot, 2005). L'absence de référence à l'histoire pourtant militante du quartier (les années 1960-1970 y virent de nombreuses luttes contre les expulsions, de la rue de l'Ouest à celles liées à la radiale Vercingétorix) en est un trait qui nous paraît significatif : les militants d'aujourd'hui ne semblent pas se situer par rapport à ce passé.

Mais à l'instar des membres de la «classe d'alternative », ces militants ne sont pas des «notabilités en puissance » : ils ne se montrent que peu intéressés par l'accès aux sphères de pouvoir politique. En revanche, ils appa- 
raissent bien davantage comme " missionnaires d'un projet sociétal », en l'occurrence un projet urbain : faire vivre ensemble toutes les populations au sein du quartier.

\section{L'espace du quartier et le discours de la mixité sociale}

Les populations « gentrificatrices » des quartiers anciens, populaires et dégradés soulignent fréquemment qu'elles apprécient le côté « exotique » et « cosmopolite » des quartiers qu'elles investissent (Bidou-Zachariasen, 1996), ou encore la « diversité », « l'authenticité d'un quartier encore populaire » (Lévy-Vroelant et alii 2005). Ces discours sont sous-tendus par une idéologie de la mixité sociale, associant un monde ouvrier plus ou moins fantasmé à des pratiques de convivialité et valorisant par là même la coexistence avec les couches populaires. Si le quartier Plaisance Pernety n'est pas suffisamment populaire - il l'est dans certains îlots mais non dans son ensemble pour se prêter à ce type de discours de la part des militants, il est néanmoins frappant de constater combien la mixité sociale est une norme forte des actions et réflexions de l'association.

Ainsi, le projet d'une pension de famille au sein du quartier est justifié $\operatorname{par}^{2}$ « le besoin de mixité sociale », laquelle, outre qu'elle est « essentielle» et non " gadget », signifie « diversifier la population, mélanger toutes les catégories sociales, diversifier les fonctions, brasser les générations ». Toutes les populations sont invitées à « vivre ensemble » dans des lieux qui doivent être créés pour cela et dont elles pourraient assumer la gestion : maison des associations, cafés associatifs, espaces culturels... La réflexion sur la mixité est approfondie par les militants qui émettent des « critiques» concernant la répartition des populations au sein de l'arrondissement (zonage du logement social et de l'accès à la propriété, emplacement du logement social...), des « propositions » pour réaliser la mixité (changer d'échelle, mixer les prêts, développer les équipements...), ainsi que des «suggestions » à la municipalité d'arrondissement en matière d'action sociale liée au logement (création d'un réseau d'intervenants, accompagnement social...).

De façon plus générale, l'association se veut active dans le développement d'une « solidarité de voisinage », à laquelle elle contribue, notamment par les fêtes qu'elle organise qui « sont des lieux de rencontre et d'échange entre les habitants », ou encore par son action militante, par exemple en faveur de l'implantation de logements sociaux « susceptibles de créer du lien entre catégories sociales par un meilleur mélange ${ }^{3} \gg$ ou d'équipements col-

2. Les citations suivantes sont issues des documents diffusés par l'association à l'occasion du montage du projet «pension de famille ».

3. Bulletin de l'association, 16 mai 2005. 
lectifs censés constituer des lieux de rencontre entre habitants. Quel que soit le répertoire d'action, festif ou militant, la volonté de nouer des liens entre couches sociales différentes constitue le leitmotiv de l'association, voire le critère par rapport auquel on pourrait évaluer la réussite des actions :

"C'était bien réussi, c'était une fête trans-milieux sociaux c'est-à-dire pas uniquement la rue Bauer Thermopyles, mais y a des gens qui sont venus des tours, d'Alésia, de tout le quartier, vraiment de très loin, de tout le quartier au sens mélange social » (homme, 40 ans, enseignant, membre du bureau de l'association).

Faire se rencontrer les habitants, tous les habitants, échanger, nouer des liens entre voisins, sont des thématiques et des objectifs essentiels. Le territoire est ici censé constituer le lien entre les différentes populations y résidant, puisque ces dernières doivent se côtoyer, se rencontrer, partager, voire faire la fête ensemble, sur la seule base de leur appartenance locale commune. Il doit être le foyer de liens sociaux, si possible interclassistes.

L'association de quartier est l'instrument de cette mission, en militant tout à la fois pour une diversification de la population et pour la création de liens entre les différentes couches sociales. Les militants, distanciés des couches populaires par leur niveau de rémunération et, dans l'ensemble, par une certaine sécurité de l'emploi, souhaitent donc tout à la fois attirer des couches populaires et nouer des liens avec elles, voire les aider : à se loger, à se loger mieux, à comprendre aussi, quand il s'agit par exemple d'aller expliquer, dans le sud de l'arrondissement, au sein des ensembles d'habitat social, les enjeux de la procédure de requalification urbaine. Le militantisme au sein de l'association de quartier paraît solidaire d'un messianisme social auquel on peut apporter plusieurs éclairages. Socialement proches des multiculturels qui investissent les quartiers populaires dégradés (Simon, 1998) mais résidant dans un arrondissement à l'image plus bourgeoise, les militants du quartier sont attachés à l'image d'un quartier « vivant » dont l'animation passe par le mélange des fonctions, mais aussi la présence de catégories populaires. Reprenant à leur compte la mythologie du quartier « convivial » d'un Paris populaire, mythologie qui n'appartient pas, la plupart du temps, à la mémoire de leur classe sociale, ces militants valorisent un mode de vie populaire plus ou moins rêvé, constitué d'ambiance festive et chaleureuse, d'échanges et d'entraide. Le maintien ou l'apport de couches populaires au sein du quartier permettrait ainsi de sauvegarder l'image d'un quartier conforme à leurs aspirations.

De surcroît, de nombreux militants manient, en tant que professionnels, un concept de «mixité sociale » devenu la norme des politiques urbaines et sociales (Schnapper, 2005). Architectes, urbanistes, travailleurs sociaux voient là une solution à tous les maux de l'urbain. Dès lors, le cadre associatif est une occasion pour eux de faire valoir ce qu'ils ne parviennent pas 
nécessairement à faire aboutir dans le cadre professionnel. Il est un instrument de transfert et d'application de leur compétence, ce qui explique, pour notre association de quartier, le degré d'élaboration du projet pension de famille et plus globalement la réflexion sur la mixité sociale. On retrouve là une caractéristique classique des militants du cadre de vie, engagés comme professionnels à l'égard des institutions étatiques et comme habitants dans des associations luttant pour (ré)orienter les politiques de ces mêmes institutions (Dagnaud, 1981).

Ainsi, les aspirations de militants soucieux d'évoluer dans un cadre de vie conforme à leurs attentes, se conjuguent avec une idéologie, transférée de leur cadre professionnel, pour expliquer la prégnance d'un discours de la mixité sociale, qui tranche nettement avec le réseau de sociabilité mis en place au sein de l'association.

\section{L'espace de l'association et la recherche de l'entre-soi}

En effet, au regard de l'importance de l'entre-soi au sein de l'association, les militants semblent davantage désireux de travailler à l'image d'un territoire qui soit conforme à l'identité sociale qu'ils désirent se voir attribuer (Benoit-Guilbot, 1986) que de créer de réels liens sociaux avec les couches populaires.

Cette recherche d'entre-soi est identifiable tout d'abord dans le choix même de l'association. Pour un certain nombre de militants, la participation aux activités d'Urbanisme et Démocratie est ainsi autant motivée par l'envie d'intégrer un collectif bien spécifique que par l'intérêt pour les affaires du quartier :

« On a été à une réunion d'ATTAC, sur le fond c'est vrai que c'était peut-être plus proche de nos préoccupations, mais en même temps c'est vrai que c'était pas la même moyenne d'âge, ça allait moins vite, c'était un autre âge qui comprenait pas forcément les choses pareil que nous, et pour nous ça a pas assez boosté et on y est pas retourné » (Femme, 25 ans, normalienne, étudiante en DEA, membre de l'association).

Cette jeune femme et son ami n'adhèrent pas à ATTAC parce qu'ils ne se sentent pas proches des militants qu'ils y rencontrent, et non parce qu'ils ne comprennent et soutiennent pas les préoccupations de cette association. Du coup, le choix d'une association ressort d'une sélection assez élitiste :

« À titre personnel c'est vrai que les gens qu'on a pu rencontrer au sein de l'association c'est aussi des gens avec lesquels on a quand même des atomes crochus, c'est une population assez jeune, d'un niveau d'études relativement élevé en général, on a rencontré des gens intéressés et intéressants et c'était plutôt motivant » (Homme, 26 ans, normalien, enseignant, membre de l'association). 
Si les actions de l'association doivent notamment viser les couches populaires, il semble que ces mêmes couches soient exclues quand il s'agit de tisser des affinités. Si tous les militants n'affirment pas le primat d'un entre-soi social et culturel de façon aussi explicite, c'est pourtant bien lui qui agit comme un filtre au sein de l'association : seuls les profils les plus congruents avec le type dominant sont cooptés par le «noyau dur».

Au fil de sélections plus ou moins implicites, le cœur actif de l'association se trouve finalement constitué d'hommes et de femmes ayant entre 25 et 35 ans, célibataires ou en couple mais sans enfant pour la plupart, diplômés (tous ont atteint le niveau de la licence) et votant plutôt à l'extrême gauche.

"Les membres de l'association c'est des copies conformes, on est tous des clones, des personnes entre 25 et 35 ans et qui votent à gauche de la gauche » (Homme, 37 ans, contrôleur aérien, trésorier de l'association).

Les conceptions socio-politiques qui soutiennent l'engagement militant sont globalement assez homogènes et marquées à gauche. Caractéristiques socio-démographiques et références politiques se renforcent pour aboutir à la création d'un collectif soudé, fonctionnant parfois comme une "bande de copains ». Les dîners à l'occasion des réunions de travail se multiplient, viennent consolider les bases du rassemblement, tout en les élargissant. Aucune réunion, d'un groupe de travail ou d'un bureau, ne se déroule sans occasionner un repas ; aucune action militante, sans se clore sur un apéritif. Fréquemment, la tenue du stand sur le marché est suivie d'un apéritif, dans un café, chez un militant, voire d'une invitation à déjeuner. Au cours de ces moments, l'importance accordée aux discussions, la satisfaction de chacun à participer, la nature des contacts entre les personnes qui s'inquiètent les unes des autres, la présence d'une mémoire commune, témoignent de la construction progressive d'un lien de type amical. L'intérêt commun aux militants n'est plus seulement l'objet de leur militance, mais également le plaisir d'être ensemble autour d'une table et de développer des rapports sociaux plus personnels. Les repas se déroulent dans une ambiance chaleureuse et permettent de tisser des liens qui vont déborder le cadre associatif : les militants vont fêter ensemble des anniversaires, s'aider lors des déménagements... Les pratiques collectives associatives sont ainsi le support de pratiques hors de ce cadre, qui vont contribuer à renforcer le caractère collectif de l'association en élargissant les bases du rassemblement.

Si ce fonctionnement présente l'avantage de faciliter la participation de ceux dont le profil permet l'intégration de l'entre-soi, il coupe de l'association des militants plus décalés :

« ...ça devenait asphyxiant... de se retrouver effectivement entre couches moyennes, entre universitaires bac +8 , y avait quelque chose de très marqué d'un point de vue socio-culturel avec une classe sociale, la nôtre... on a fini par trouver que l'association fonctionnait plutôt comme une bande de copains, un 
peu fermée, bande de copains à laquelle on n'appartenait pas d'ailleurs, et nous on voulait pas que ce soit une sorte de groupuscule » (Femme, 40 ans, enseignante, membre de l'association).

Le problème peut se régler par le départ des « décalés » ou des « indésirables ». Plus problématique, du point de vue de l'action de l'association, est le risque de couper cette dernière de sa «mission » de mise en lien avec le quartier :

«...si on fait trop bande de copains, c'est pas bon non plus pour intégrer les gens du quartier, ou on intégrera qu'une certaine partie des gens du quartier, mais c'est toujours con de se retrouver entre gens qui pensent la même chose » (Homme, 37 ans, contrôleur aérien, trésorier de l'association).

Quelques années (5 ans) après la réalisation de ces entretiens, l'association n'a pas intégré de gens du quartier appartenant à des couches populaires, même si les fêtes organisées quatre fois par an rassemblent un éventail assez large de population. Mais c'est paradoxalement parce qu'elle s'est constituée comme un entre-soi socio-culturel qu'elle est parvenue à faire valoir la validité d'un projet dont «l'objectif » est justement de « réintroduire la mixité sociale au sein du quartier ». Outre le fait que le rassemblement de compétences permet à l'association de constituer un dossier solide pour l'obtention du droit de gestion d'une «pension de famille », elle fait valoir un entre-soi qui la crédibilise (le projet a reçu l'aval des autorités compétentes) comme future gestionnaire de la structure : « Sans être des professionnels de la gestion locative sociale, les 37 membres fondateurs de l'association Pension de famille à Bauer-Thermopyles-Plaisance réunissent un grand nombre de compétences. Ils exercent les métiers suivants [...] ». Il s'ensuit une liste au sein de laquelle on peut compter huit personnes exerçant des fonctions intermédiaires ou d'encadrement dans l'action sociale et la santé, sept personnes liées à l'enseignement, à la recherche, à l'architecture et à l'urbanisme, quatre personnes évoluant dans le monde des médias et de la culture, des ingénieurs et techniciens, un avocat, une consultante ressources humaines.

Le recours au territoire permet à ces héritiers de la «nouvelle classe » de se poser en « entrepreneurs de morale » (Becker, 1963) tout en ménageant leurs propres intérêts. Le mélange des milieux sociaux sur un même territoire est posé comme un intérêt sociétal universel, intérêt dont les militants sont porteurs, au nom duquel ils agissent et prescrivent ce qui doit être fait. Ce faisant, ils travaillent à préserver ou infléchir l'image de leur territoire dans un sens conforme à leurs aspirations. Cependant, dans leurs pratiques, le territoire n'est pas celui de tous les liens sociaux. Si le territoire est censé être le lieu de tous les liens, il en est aussi entre lesquels les liens ne s'établissent pas malgré leur appartenance à un même territoire, ou alors de façon occasionnelle et temporaire. 


\section{QUAND LE TERRITOIRE FAIT LIEN MALGRÉ TOUT}

Nous avons identifié deux moments, impulsés par l'action de l'association de quartier, au cours desquels le territoire se fait véritablement le lieu des liens puisque c'est sur la base de la seule appartenance territoriale que des liens sociaux s'établissent, et s'établissent plus spécifiquement entre couches sociales différentes.

\section{Le territoire ludique}

L'observation des fêtes de quartier organisées par l'association permet de constater l'importance des liens de voisinage pour les citadins, tout d'abord au travers de leur succès en termes d'affluence, ensuite par les modalités selon lesquelles se déroulent les échanges. Autour des « ateliers » (peinture murale, maquillage, contes, réparation de vélos, etc...), ou des « stands » de boissons ou de nourriture, les habitants se saluent et se parlent. Les échanges sont, la plupart du temps, articulés autour du quartier : les discussions évoquent les événements notables, les commerçants, les lieux appréciés... Ils s'initient et se développent aussi facilement - du moins plus facilement que ce que la co-présence dans l'espace public induit de façon habituelle - parce que la co-présence atteste, ici, d'un lieu de résidence commun. Outre le fait que cette communauté de lieu d'habitat facilite la prise de contact ( « vous habitez où dans le quartier? »), elle réduit la distance existant entre les citadins puisqu'elle induit un partage de lieux, qui sont aussi des lieux de vie.

Ce que les habitants manifestent en venant aux fêtes dépasse ainsi le simple plaisir de passer un moment agréable. Il s'agit d'un attachement au quartier qui est un attachement aux formes spatiales mais surtout aux formes de vie sociales qui s'y sont développées :

« Quand je suis arrivé dans le quartier, je l'ai trouvé vivant, surtout vu là d'où je venais, c'était mort ; là y a des commerces, ça bouge, en même temps c'est pas comme dans le VIII où y a plein de monde la journée et puis le dimanche matin tu peux faire deux kilomètres sans croiser personne » (Homme, 30 ans, chercheur, membre de l'association).

« J'aime bien le quartier, boire mon petit café sur le zinc au bistrot du coin » (Homme, 35 ans, habitant le quartier, lors d'une fête organisée par l'association).

Le partage d'un lieu est aussi celui des pratiques liées à sa morphologie, ses équipements collectifs...La fête de quartier est un moment où tous s'affirment et par conséquent se reconnaissent comme habitants de ce quartier, c'est-à-dire des individus partageant un certain nombre de valeurs communes liées à la vie dans ce quartier. Ce partage, en réduisant l'étrangeté mutuelle 
des citadins (Simmel, 1908) permet d'instaurer un lien avec l'autre, voisin désormais identifié et reconnu comme tel.

Il ne s'agit pas là de créer des relations durables au-delà de l'instant festif, mais de donner corps et substance à une identité territoriale, de rendre vivant un lien de proximité qui permet d'éviter un anonymat jugé dommageable à la vie dans les grandes villes. La plupart du temps, les liens développés dans le cadre de ces fêtes restent sans suite : ils se limitent à des échanges ponctuels sur le quartier et sur le commun attachement à cet espace. Ils forment cependant le support de ces courts échanges et signes de reconnaissance qui auront lieu par la suite lorsque se croiseront, au coin d'une rue, des participants à la fête. Suite à l'organisation de la première fête de quartier situé sur un terrain en friche, les principaux responsables de l'association évoquent, avec un étonnement et un plaisir non dissimulé, le fait d'être à présent « reconnus » quand ils marchent dans la rue. Au cours d'un entretien sur ses motivations à l'engagement, une jeune « recrue » évoque le même sentiment :

« Tu marches dans le quartier, tu dis bonjour à des gens que tu as vus, avec lesquels tu as manifesté, tu as bu un café, et ça je trouve ça sympa » (Femme, 24 ans, étudiante à l'École des Chartes, membre de l'association).

Le territoire informe très largement ces liens entre voisins. Il est au cœur des échanges et des conversations, mais surtout il est ce que les participants partagent et qui leur permet d'aller les uns vers les autres. À ce titre, il est le support d'un rassemblement plus large que ce que la différenciation sociale des pratiques autorise habituellement. La fête de quartier est une occasion pour que se réduisent temporairement un certain nombre de distances, sur la base d'une appartenance au quartier qui est, elle, commune à tous les habitants. Il n'est, dès lors, pas rare d'assister à d'improbables scènes, tel ce vieux monsieur d'un immeuble bordant le lieu de la fête qui ouvre sa fenêtre pour faire entendre un disque d'accordéon. Il descendra ensuite raconter le quartier tel qu'il l'a connu quand il était jeune. L'espace urbain se fait ici le support de rapports sociaux apparemment pacifiés puisqu'à l'ignorance mutuelle de populations d'origines sociales ou d'âges différents se substituent des échanges sociaux qui trouvent une justification dans le partage d'une même identité territorialisée. La co-présence à la fête $d u$ quartier, en rendant effective cette identité locale, autorise à dépasser le quant-à-soi ordinaire pour aller vers l'autre... le temps d'une fête.

Ces échanges sociaux éphémères se déroulent ainsi dans une ambiance que l'on pourrait qualifier d'enchantée, en raison de cette abolition temporaire de distances générationnelles ou sociales qui guident habituellement les relations sociales, ainsi que de l'atmosphère joyeuse dans laquelle elles baignent. Il s'agit bien de faire la fête et les modalités même de l'organisation facilitent les échanges : chacun est en effet invité à apporter chaises, tables, 
nourriture, boissons... Les habitants viennent eux-mêmes dans un état d'esprit qui les porte à s'adresser facilement la parole. La commensalité joue son rôle habituel d'instrument du lien social en permettant d'associer circulation des mets et du verbe. L'alcool permet à quelques langues de se délier plus facilement et facilite les contacts. Un groupe de musique est fréquemment invité à se produire et il arrive que la fête, plus particulièrement au mois de juin, se termine en bal. Les associations ont bien saisi l'impact de ces instants festifs sur la vie sociale au sein du quartier, et le terrain vague sur lequel ils se déroulent n'est pas choisi par hasard. La copropriété qui le détient est menacée d'expulsion par la Ville de Paris qui veut en faire un terrain constructible. En y organisant des événements, l'association en fait le lieu du lien par excellence, ce qui facilitera effectivement la mobilisation des habitants afin de défendre ce qui est devenu un symbole de solidarité locale.

\section{Le territoire revendicatif}

Il arrive ainsi que le festif se mêle au militant, qui est lui-même créateur de liens. Dès lors que le quartier constitue un espace de référence pour ses habitants, ces derniers sont susceptibles d'être amenés à se regrouper pour le " défendre ». Les associations attachées au cadre de vie local naissent ainsi la plupart du temps en réaction à un projet d'aménagement qu'elles rejettent, dans son contenu et/ou dans des modalités de réalisation jugées insuffisamment participatives ou concertées. Dès lors, des actions plus ou moins conventionnelles et illégales vont être décidées : signatures de pétitions, blocages de chantier, occupation de permanence d'élus, détournement de conférences de presse etc... Leur caractère collectif est important pour en permettre le déroulement, ainsi que pour attirer l'attention des médias et se constituer comme force de pression face aux institutions, mais il ne faut pas surestimer l'importance. Les personnes effectivement mobilisées sont la plupart du temps peu nombreuses et les actions sont considérées comme des " réussites » si elles parviennent à mobiliser, lors d'une manifestation par exemple, entre 50 et 100 habitants. L'espace du quartier, même s'il est aujourd'hui un lieu auquel les citadins sont attachés, notamment dans les centres anciens (Authier, 2001), n'est pas le support le plus mobilisateur pour des actions collectives militantes. Mais ces dernières nous intéressent ici moins en elles-mêmes que parce qu'elles sont l'occasion de nombreuses interactions qui mettent en relation les membres de l'association et d'autres habitants du quartier.

À l'occasion des blocages de chantier, les militants se rassemblent au petit matin devant le lieu à défendre et dégustent ensemble café et croissants apportés par chacun. Les habitants discutent sur le trottoir, se lancent dans des échanges sur le sens de l'action qu'ils sont en train de mener ou se 
demandent plus simplement des nouvelles de leurs proches. Certains ne font que passer, disent bonjour en allant au travail, en emmenant les enfants à l'école. D'autres terminent le blocage du chantier en allant boire un café. Les liens militants donnent ici naissance à une sociabilité amicale qui, en se déployant à la vue de tous, comporte un certain pouvoir d'attraction sur les passants. Il arrive ainsi que ces derniers, étonnés par ces surprenants attroupements, s'enquièrent de ce qui est en train de se dérouler et se joignent aux discussions. Il s'agit là du pendant militant des relations « enchanteresses » développées dans le cadre des fêtes, quand le citadin dépasse sa réserve habituelle pour aller à la rencontre d'un autre.

Le militantisme soutient ainsi des interactions qui concernent le quartier comme réalité spatiale et sociale. Réalité spatiale, car ces actions ont pour cible des aménagements prévus dans le quartier. Le devenir des formes du quartier est au cœur des liens que les militants nouent avec les habitants. Réalité sociale, car les actions militantes ne concernent pas uniquement la seule population associative, mais elles mettent au contraire les membres de l'association en lien avec l'ensemble des composantes sociales du quartier, notamment les couches plus populaires. L'une des batailles de l'association a ainsi consisté à préserver le " château ouvrier », un ancien couvent vendu en 1905 à un riche propriétaire qui décide d'y installer 49 logements pour des gens aux revenus modestes. En 1998, il reste 21 occupants habitant l'immeuble depuis plus de 30 ans, occupants qui doivent être expulsés dans le cadre de la ZAC Didot. Un autre chantier de l'association est la mise en place et la gestion d'une résidence sociale à destination de familles en situation précaire. Dans le cadre de ces différents " dossiers », les militants prennent contact avec les habitants concernés, s'informent de leurs conditions de vie, leur positionnement par rapport aux aménagements prévus. Il arrive que les actions menées concourent à la prise en compte des intérêts représentés par l'association, et le « château ouvrier » sera par exemple préservé et réhabilité. Mais les interactions ne débouchent pas, la plupart du temps, sur de véritables relations avec le public « cible » de l'action. Elles mettent néanmoins en lien des populations qui habituellement ne s'abordent pas, sur la base d'une commune appartenance et surtout d'un attachement au quartier entendu comme forme indissociablement sociale et spatiale qu'il s'agit de «préserver».

La tenue du stand sur le marché le dimanche matin peut également donner naissance à de ponctuelles rencontres, lorsqu'un passant interpellé s'intéresse aux activités de l'association et s'informe des actions menées. Les interactions sont alors construites sur le même mode que celles qui se déroulent par exemple lors d'un blocage de chantier : le citadin sort de sa réserve pour aller vers l'autre, sur la base de son appartenance au quartier et d'un éventuel attachement aux dispositifs menacés ou qui risquent de l'être dans 
l'avenir. Il n'est pas rare que des personnes âgées s'arrêtent pour bavarder. Habitant le quartier depuis longtemps, elles font part de leur expérience et de leur opinion concernant les dossiers en cours. Il arrive également que les interactions soient conflictuelles. Alors que l'association élabore une « carte du bruit » dans l'arrondissement et la présente sur le marché, des passants interpellés pour donner leur avis expriment leur incompréhension face à un dossier qui leur paraît comporter moins d'intérêt que d'autres causes plus « sociales ». Les militants sont alors renvoyés plus ou moins brutalement à la défense de leur « pas de porte » qui, seule, les préoccuperait. Les divergences d'intérêt qui se manifestent lors de ces interactions viennent souligner que ces dernières, en permettant la confrontation de points de vue différents, expriment la réalité sociale du quartier qui déborde la représentation qu'en donne l'association.

Ces liens, festifs ou militants, existent, mais ils ne dépassent pas l'événement qui les a suscités. La plupart du temps, les relations ainsi initiées sont éphémères. Elles n'en sont pas moins fortement structurées par l'espace local du quartier.

C'est tout d'abord une identité commune d'habitant du quartier qui soutient ce mouvement vers l'autre, concrétisé par un signe de reconnaissance, un bref échange verbal, même conflictuel. C'est parce que l'on se reconnaît comme habitant du quartier que l'on se sent en droit de le défendre, de s'opposer à ceux qui croient le défendre ou de fêter cette appartenance. La mutuelle reconnaissance de cette identité est basée sur une communauté de lieu qui est aussi, dans une certaine mesure, une communauté de valeurs portées par ce lieu. Les habitants qui assistent aux fêtes de quartier ou qui participent aux actions militantes sont réunis par un commun attachement aux formes de vie sociales qui s'y sont développées, attachement qui touche de plus larges composantes de la population que les seuls militants, notamment des membres des classes populaires ancrées depuis longtemps dans le quartier.

Le quartier est ensuite l'objet de toutes les interactions, plus particulièrement de toutes les conversations nouées dans le cadre des événements festifs ou militants : il est ce dont on discute ou débat ; il est aussi ce que l'on fête. L'espace se fait ici fondamentalement le lieu des liens, leur raison d'être.

Il est enfin le support des liens car il est la réalité sociale et spatiale spécifique sur laquelle ces derniers reposent. Qu'il s'agisse des interactions festives ou militantes, elles mettent aux prises les différentes composantes du quartier, habitants vieux et jeunes, couches moyennes et couches populaires. Nous les avons qualifiées «d'enchantées » pour exprimer cette transcendance, ponctuelle et non habituelle, de distances s'exprimant plus couramment par des pratiques différentes au sein du quartier. Les formes spatiales sont ici le support d'interactions intrinsèquement sociales puisqu'elles sont ce qui permet, un moment, de faire société. 


\section{Conclusion}

Le territoire est bien le support de liens sociaux qui font exister le quartier comme forme de vie sociale, liens festifs et militants, entre habitants du quartier par ailleurs peu habitués à se fréquenter, étant donné le fractionnement social des pratiques au sein de l'espace urbain. Cette sociabilité de voisinage est occasionnelle et ponctuelle, elle ne débouche pas sur la création de relations plus durables entre les habitants. Elle est cependant l'occasion, notamment pour les militants de l'association de quartier, d'entrer en contact avec des couches sociales plus populaires et de les mettre ainsi en accord avec les discours de l'ouverture et de la mixité sociale qu'ils professent, discours qui structurent une grande partie de leur pratique associative, mais aussi leur regard sur le territoire de la ville.

Pour ces militants héritiers de la «classe d'alternative » identifiée dans les années 1970 et 1980, le territoire est l'objet d'un double discours, ou plus exactement d'un discours entrant en contradiction avec leurs pratiques : en reprenant à leur compte la norme éthique de la mixité sociale, ils font du territoire le support d'un idéal de sociabilité, et plus largement d'un idéal de société, qu'ils concourent à déconstruire en pratiquant activement l'entre-soi, notamment dans le cadre associatif. Or, cette recherche d'entre-soi contribue, justement, à faire fuir les autres que soi, selon des mécanismes bien identifiés (Dansereau, 2000 ; Simon, 1998). Le territoire est alors davantage un prétexte au déploiement d'un discours normatif participant de la constitution d'une classe sociale, que le support de liens sociaux qui restent guidés par l'origine et l'appartenance sociales.

\section{RÉFÉRENCES BIBLIOGRAPHIQUES}

AuthIER, J.-Y. 2001. « Les rapports aux quartiers » dans J.-Y. Authier (sous la dir. de), Du domicile à la ville. Vivre en quartier ancien, Paris, Anthropos, p. 133169.

BACQUÉ, M.-H. ; Fol, S. ; LÉvY, J.-P. 1998. « Mixité sociale en banlieue ouvrière : enjeux et représentations », dans N. Haumont et J.-P. Levy, La ville éclatée. Quartiers et peuplement, Paris, L'Harmattan, p. 161-173.

BECKER, H. 1963. Outsiders, Éditions Métailié, Paris.

Benoit-Guilbot, O. 1986. «Quartiers-dortoirs ou quartiers-villages » dans L'esprit des lieux, Programme OCS, CNRS, Paris, p. 127-156.

BIDART, C. 1988. « Sociabilités : quelques variables », Revue française de sociologie, vol. $29, \mathrm{n}^{\circ} 4$, p. 621-648.

BIDOU-ZACHARAZIEN, C. 1996. "Classes populaires et classes moyennes en centre historique rénové », dans $\mathrm{N}$. Haumont, La ville : agrégation et ségrégation sociales, Paris, L'Harmattan, p. 131-142. 
Chalvon, S. ; Claverie, E. 1984. « Le triangle du XIV siècle » Archives de l'ocs, 1979, vol. 2, p. 26-53.

Cherki, E. ; MeHL, D. 1979. Les nouveaux embarras de Paris. De la révolte des usagers des transports aux mouvements de défense de l'environnement, Paris, Maspero.

Dagnaud, M. 1979. La banlieue autrement. La vie sociale à Cergy-Pontoise, Paris, Fondation des villes.

Dagnaud, M. 1981. «La classe "d'alternative". Réflexion sur les acteurs du changement social dans les sociétés modernes », Sociologie du travail, $\mathrm{n}^{\circ} 4$, octobredécembre,

DANSEREAU, F. 2000. « Montréal : ségrégation socio-résidentielle et cohabitation sociale » dans F. Dureau, V. Dupont, E. Lelièvre, J.-P. Lévy, T. Lulle (sous la dir. de), Métropoles en mouvement, Paris, Economica, p.189-297.

HÉrAN, F. 1987. « Les relations de voisinage », Données sociales, INSEE, p. 326-337.

HÉRAN, F. 1988. « La sociabilité, une pratique culturelle », Économie et Statistique $\mathrm{n}^{\circ} 216$, INSEE.

ION, J. ; Micoud, A. ; NizEY, J. 1979. Associations résidentielles et institution municipale : le cas de Saint-Étienne, Rapport de recherche, Saint-Étienne, CRESAL.

Ion, J. ; Gillio, C. 2001. "Point de vue sur une consultation de recherche I Dynamique du cadre de vie et actions associatives », Actions associatives, solidarités et territoires, Actes du colloque, Saint-Étienne, 18-19 octobre, p. 39-49.

Ion, J. ; Franguiadakis, S. ;ViOT, P. 2005. « Militer aujourd'hui » Éditions Autrement, Cevipof, Paris.

Lévy-Vroelant, C. ; Dussart, B. ; Frey, J.-P. 2005. « Entre paradoxale transparence et impossible entre-soi »La société des voisins. Partager un habitat collectif dans B. Haumont et A. Morel (sous la dir. de), DAPA, collection Ethnologie de la France, Cahiers 21.

Ment, D. 1982. «Culture et action associatives », Sociologie du travail, $\mathrm{n}^{\circ} 1$, p. $24-42$.

SCHNAPPER, D. 2005. «Idéal et limites de la mixité sociale. Les arguments du débat public », Informations sociales, $\mathrm{n}^{\circ} 125$.

SIMMEL, G. 1908. «Digressions sur l'étranger » dans Y. Grafmeyer et I. Joseph, 1990. L'École de Chicago. Naissance de l'écologie urbaine, Paris, Aubier, p. 53-59.

SimON, P. 1998. «L'intégration au quartier à l'épreuve de la rénovation », dans N. Haumont ; J.-P. Lévy (sous la dir. de), La ville éclatée. Quartiers et peuplement, Paris, L'Harmattan.

VERMEERSCH, S. 2002. «Le sens de l'engagement associatif : logiques individuelles et logiques sociales de l'action », Thèse sous la direction de B. Haumont, Université de Paris 10, décembre 2002.

VERMEERSCH, S. 2004. «Entre individualisation et participation : l'engagement associatif bénévole », Revue française de sociologie, 45-4, 681-710. 